Original article

\title{
The occurrence of vulvovaginal Candida species and their antifungal susceptibility pattern in HIV seropositive women in Ahvaz, Southwest Iran
}

\author{
Mehran Varnasiri $^{\mathrm{a}}$, Shokrollah Salmanzadeh ${ }^{\mathrm{a}}$, Ali Zarei Mahmoudabadi ${ }^{\mathrm{a}, \mathrm{b}}$, \\ Marzieh Halvaeezadeh $^{\mathrm{b}}$, Simin Taghipour ${ }^{\mathrm{b}}$, Shahla Molavi ${ }^{\mathrm{c}}$, Seyed Mohammad Alavi ${ }^{\mathrm{a}, * *}$, \\ Khojasteh Hoseiny Nezhad ${ }^{\mathrm{d}}$, Parastoo Moradi Choghakabodi ${ }^{\mathrm{d}, \mathrm{e}}$ \\ ${ }^{a}$ Infectious and Tropical Diseases Research Center, Health Research Institute, Ahvaz Jundishapur University of Medical Sciences, Ahvaz, Iran \\ ${ }^{\mathrm{b}}$ Department of Medical Mycology, School of Medicine, Ahvaz Jundishapur University of Medical Sciences, Ahvaz, Iran \\ ${ }^{\mathrm{c}}$ Health Psychology, Ahvaz Jundishapur University of Medical Sciences, Ahvaz, Iran \\ ${ }^{\mathrm{d}}$ Physiology Research Center and Department of Physiology, Ahvaz Jundishapur University of Medical Sciences, Ahvaz, Iran \\ e Thalassemia and Hemoglobinopathy Research Center, Health Research Institute, Department of Emergency Medicine, Ahvaz Jundishapur University of Medical Sciences, \\ Ahvaz, Iran
}

\section{A R T I C L E I N F O}

\section{Keywords:}

Vulvovaginal Candidiasis

HIV

Amphotericin B

Itraconazole

Caspofungin

\begin{abstract}
A B S T R A C T
Background: Vulvovaginal candidiasis (VVC) is a prevalent infection among HIV-seropositive women that still needs to be subjected to more researches. This study is aimed at determining the occurrence of vulvovaginal Candida species and their antifungal susceptibility pattern in HIV-seropositive women from the Behavioral Disease Counseling Center in Ahvaz, Iran.

Method: In this analytical cross-sectional study, $85 \mathrm{HIV}$-seropositive women with the mean age of 26.15 years (SD. 9.60) were evaluated. Vaginal swabs were collected from women to identify various Candida species through conventional methods and API 20 C AUX system. Antifungal susceptibility testing was done on specimens against Amphotericin B and Azole antifungals. Eventually, all data were analyzed by SPSS.

Results: The occurrence of vulvovaginal Candida species was 34.11\% (29) among 85 HIV-seropositive women. Out of 29 Candida spp., $18(62.06 \%)$ C. albicans, 6 (20.68\%) C. glabrata, 4 (13.79\%) C. dubliniensis, and 1 $(3.44 \%)$ C. krusei were identified. There was no significant association between VVC and some medical variables such as antiretroviral therapy, birth control methods, underlying diseases, and CD4 count (PV $>0.05$ ). However, there was a significant association between VVC and age and pregnancy (PV $<0.05$ ). The most Candida spp. showed considerably resistant to Amphotericin B and Itraconazole while all species were susceptible to Caspofungin.

Conclusion: The high occurrence of Candida spp. (34.11\%) among HIV-seropositive women is notable, with the highest frequency of C. albicans, C. glabrata and C. dubliniensis, respectively. Also, their notable resistance to Amphotericin B and Azole antifungals necessitates more effective treatments for VVC, particularly in pregnant women.
\end{abstract}

\section{Introduction}

Vulvovaginal candidiasis (VVC) is the most prevalent gynecological infections among childbearing women. ${ }^{1}$ The frequency of vaginal Candida spp. was significantly higher among HIV-seropositive women than HIV-seronegative women. ${ }^{2}$ Mucosal candidiasis (vaginal, oropharyngeal, and esophageal) is one of the most common infections among HIV-infected patients. ${ }^{3-5}$ Various behavioral and biological risk factors may predispose individuals to mucosal candidiasis. ${ }^{6}$ For example, risk factors including high sexual activity, pregnancy, diabetes, and or using antibiotics can arise the risk of vaginal candidiasis. ${ }^{7,8}$ Moreover, cell-mediated immunodeficiency increases the risk of mucosal Candida colonization and candidiasis. ${ }^{8,9}$ The spectrum of Candidiasis is varied from asymptomatic colonization to symptomatic form. The low CD4 T-lymphocyte count has previously been shown as the main risk factor for the development of mucosal candidiasis. ${ }^{10}$ Despite the therapeutic effects of antiretroviral therapy (ART) on the AIDS-related infectious, ${ }^{11}$ Candidiasis still remains a common opportunistic infection in HIV seropositive patients, ${ }^{12}$ mostly because of increasing antibiotic resistance. The high prevalence of candidiasis $(\geq 50 \%)$ was

\footnotetext{
* Corresponding author.

E-mail address: alavi1329dr@yahoo.com (S.M. Alavi).
} 
reported among HIV positive patients in different regions, ${ }^{13-16}$ and $C$. albicans as the most common species. ${ }^{10,13,16,17}$

Based on the researches in different regions of Iran, the prevalence of VVC and Candida spp. have been reported, for example Kermanshah (20\%), Mazandaran (28.2\%), Esfahan (54.6\%), and Tehran (87.5\%), respectively. ${ }^{18-21}$

However, few data are available regarding the rates of vulvovaginal candidiasis and or Candida species among HIV-infected patients, especially from Iran. Furthermore, the increasing global risk of antibioticresistant infections particularly azole-resistant Candida species ${ }^{22,23}$ necessitate assessing their antifungal susceptibility pattern in $H I V$-infected patients. So, the present study report the occurrence of vulvovaginal Candida species and their antifungal susceptibility pattern in HIV-seropositive women from the Behavioral Disease Counseling Center in Ahvaz, Iran.

\section{Materials and methods}

In this descriptive-analytic and cross-sectional study, $85 \mathrm{HIV}$-seropositive women with the age range between 16 and 60 years $(26.15 \pm 9.60)$ were evaluated, who were referred to the Behavioral Disease Counseling Center in Ahvaz. This study was approved by the ethics committee of Ahvaz Jundishapur University of Medical Sciences with Ethical Code: ajums.REC.1396.410. The informed consent forms were signed by all participants after explaining its contents.

Vaginal swab specimens were collected from women to identify various Candida species through conventional methods including culturing on the mediums of CHROMagar Candida or Cornmeal agar plus $\% 1$ Tween 80, Germ tube test and API 20 C AUX system.

\section{Vulvovaginal Candida species}

Three vaginal swabs were taken from the posterior vaginal fornix, and one of them was used for direct smear examination, and the others were aerobically inoculated on sabouraud dextrose agar (SDA; Zist Kavosh Iranian Company, IRAN) at $37^{\circ} \mathrm{C}$. In order to examination of vaginal candidiasis swabs, $10 \%$ Potassium hydroxide preparation $(\mathrm{KOH}$ preparation; Zist Kavosh Iranian Company, IRAN), Gram staining as well as microscopic evaluation were used. The second swab was cultured into SDA (Zist Kavosh Iranian Company, IRAN) and SDA with chloramphenicol (Zist Kavosh Iranian Company, IRAN), and then incubated for $48 \mathrm{~h}$ at $27^{\circ} \mathrm{C}$. The pure colonies were isolated and confirmed on Gram staining. For detecting Candida spp., isolated Candida colonies were subcultured onto differential CHROM agar Candida medium for $24 \mathrm{~h}$ at $37{ }^{\circ} \mathrm{C}$ (based on colony color of growing Candida spp.).

\subsection{Candida Strains}

The API $20 \mathrm{C}$ AUX system (Bio merieux, France) was used for identification and confirmation of Candida species (based on manufacturer guidelines of kit). The strips were read after incubation at $30{ }^{\circ} \mathrm{C}$ for 48-72 h. Based on this method, C. albicans (green colonies), C. $d u-$ blinensis (dark green colonies), Candida glabrata (purple colonies), Candida krusei (pink colonies) were detected.

2. The association between medical variables and vulvovaginal candidiasis

The characteristics and some medical variables of women were evaluated, including the usage history of antibiotics, antiretroviral therapy, history or presence of underlying diseases such as diabetes, CD4 count (less or greater than 200 cells $/ \mathrm{mm}^{3}$ ) and duration of AIDS.

\section{Antifungal susceptibility test}

Antifungal susceptibility testing was done on specimens against various antifungals by broth microdilution assay and according to M27 A3 CLSI protocol. ${ }^{24}$ An overnight culture of isolates was prepared in Sabouraud dextrose agar (Merck, Germany). A suspension of each isolate prepared and was adjusted to $0.5 \mathrm{McF}$ arland standard. A $100 \mu \mathrm{L}$ of each serial dilution of antifungals including, amphotericin B (Sigma Aldrich, Germany) (16-0.062 $\mu \mathrm{g} / \mathrm{mL}$ ), caspofungin (Sigma - Aldrich, Germany) (4-0.031 $\mu \mathrm{g} / \mathrm{mL}$ ), itraconazole (Sigma - Aldrich, Germany) $(16-0.125 \mu \mathrm{g} / \mathrm{mL})$ and fluconazole (Serva, USA) $(64-0.5 \mu \mathrm{g} / \mathrm{mL})$ was inoculated into each well in 96 wells microplate. Then, $100 \mu \mathrm{L}$ of diluted suspension was added into each well and incubated at $35{ }^{\circ} \mathrm{C}$ for 24-48 h. Minimum inhibitory concentration (MIC) rage, $\mathrm{MIC}_{50}, \mathrm{MIC}_{90}$ and $\mathrm{MIC}_{\mathrm{GM}}$ were calculated.

\section{Data analysis}

All data were analyzed using SPSS ver. 22. The data related to qualitative and quantitative variables were respectively reported as frequency or percentage, and mean \pm standard deviation.

The primary assessment normal distribution of variables by Kolmogorov-Smirnov Test showed that none of them follows a normal distribution. So, the results and assumptions were eventually analyzed by Mann-Whitney $U$ test and Chi-Square test with considering $\mathrm{PV}<0.05$ as statistically significant.

\section{Results}

The occurrence of vulvovaginal Candida species was $34.11 \%$ (29) among 85 HIV seropositive women. Out of 29 Candida spp., 18 $(62.06 \%)$ C. albicans, $6(20.68 \%)$ C. glabrata, 4 (13.79\%) C. dubliniensis, and $1(3.44 \%)$ C. krusei were identified. There was no significant association between vulvovaginal candidiasis and some medical variables, including the usage history of antibiotics, antiretroviral therapy, birth control methods, history or presence of underlying diseases such as diabetes, CD4 count (less or greater than 200 cells $/ \mathrm{mm}^{3}$ ) and duration of AIDS [Table 1].

However, there was a significant association between vulvovaginal candidiasis and age and pregnancy (PV $<0.05)$. In this regard, the mean age of $H I V$-infected women with candidiasis $(24.68 \pm 8.99)$ was considerably less than women without candidiasis (33.96 \pm 15.59). The most Candida spp. showed considerably resistant to Amphotericin $\mathrm{B}$ and Itraconazole while all species showed antifungal susceptibility to Caspofungin. Although, C. dubliniensis showed dose-dependent resistance to Itraconazole after $48 \mathrm{~h}$ growing, it was absolutely resistance after $72 \mathrm{~h}$ growing (Fig. 1).

\section{Discussion}

Many studies have reported the prevalence of candidiasis among women from different regions. Nurat et al.'s study reported that the prevalence of VVC was $25 \%$ among asymptomatic pregnant women in Nigeria. In this regard, the prevalence of candidiasis in women in the age range of 20-29 years (33.8\%) was more than those in the age range of 30-39 years (24.3\%). ${ }^{17}$ In another study, the prevalence of VVC was reported $30 \%$ in Nigeria ${ }^{25}$ and $30.7 \%$ in Jamaica. ${ }^{26}$

Beigi et al.'s study revealed that asymptomatic VVC was about $5-30 \%$ in Ohio, USA. ${ }^{27} \mathrm{~A}$ high rate of VVC was reported in women in the age range of the 20-29 years in many studies, ${ }^{17,28}$ which was consistent with our findings $(24.68 \pm 8.99$ years), probably due to drug usage and or contraceptives. Spinillo et al. reported a high rate of Candidiasis among married women between 30 and 45 years with frequent sexual activity, reproductive history, and taking contraceptives. $^{29}$ Our findings showed that the rate of pregnancy is significantly more frequent among women with VVC. In this regard, Dias et al.'s study was reported the frequency distribution of $92.3 \% \mathrm{C}$. albicans, $3.3 \%$ C. krusei, $2.2 \%$ C. glabrata, $1.1 \%$ C. parapsilosis and $1.1 \%$ 
Table 1

The statistically association between vulvovaginal candidiasis and some medical variables.

\begin{tabular}{|c|c|c|c|}
\hline Medical Variables & $\begin{array}{l}\text { HIV-infected women with candidiasis } \\
(\mathrm{n}=29)\end{array}$ & $\begin{array}{l}\text { HIV-infected women without candidiasis ( } n=29 \text {, randomly } \\
\text { selected) }\end{array}$ & P Value \\
\hline Duration of AIDS (month) & $29.17 \pm 17.59$ & $31.34 \pm 16.79$ & $0.5646^{\text {Mww }}$ \\
\hline AGE (year) & $24.68 \pm 8.99$ & $33.96 \pm 15.59$ & $0.0298^{\text {MWW }_{*}}$ \\
\hline CD4 > 200 cells $/ \mathrm{mm}^{3}$ & $430.68 \pm 364.69$ & $455.86 \pm 377.15$ & $0.9566^{\text {Mww }}$ \\
\hline CD4 $\leq 200$ cells $/ \mathrm{mm}^{3}$ & $424.65 \pm 359.39$ & $433.96 \pm 364.49$ & $0.8825^{\text {MWw }}$ \\
\hline \multicolumn{4}{|l|}{ The usage history of } \\
\hline Antibiotics & $17(58.62 \%)$ & $22(75.86 \%)$ & $0.1619^{c}$ \\
\hline Birth control pills & $2(6.89 \%)$ & $4(13.79 \%)$ & $0.3885^{\mathrm{c}}$ \\
\hline Antiretroviral therapy & $20(68.96 \%)$ & $25(86.20 \%)$ & $0.1154^{\mathrm{c}}$ \\
\hline - Contraception (Birth control methods) & $16(55.17 \%)$ & $17(58.62 \%)$ & $0.7909^{c}$ \\
\hline \multicolumn{4}{|l|}{ History or presence of } \\
\hline _Underlying diseases & $5(17.24 \%)$ & $9(31.03 \%)$ & $0.2197^{\mathrm{c}}$ \\
\hline _Pregnancy & $17(55.17 \%)$ & $1(3.44 \%)$ & $<0.0001^{\mathrm{c} * * *}$ \\
\hline Diabetes & $4(13.79 \%)$ & $2(6.89 \%)$ & $0.3885^{\mathrm{c}}$ \\
\hline
\end{tabular}

MWW: Mann-Whitney $U$ test.

C: Chi-square test.

$* \mathrm{PV}<0.05$ is considered as statistically significant value.

C. tropicalis among pregnant women with VVC in Brazil. ${ }^{30}$ Moreover, other studies were reported $C$. albicans as the most prevalent isolate from VVC in HIV-infected and uninfected women (80-90\%). ${ }^{31,32}$

Oliveira et al. reported a higher frequency of Candida spp. among $H I V$-infected women (29.7\%) than controls (14.5\%) in Brazil. Based on their findings, C. albicans was the most frequent isolated species in both $H I V$-infected $(52.3 \%)$ and uninfected women (85.7\%), followed by $C$. parapsolis in $17.6 \%$ and $14.3 \%$, respectively. However, the frequency of Candida spp. colonization and VVC were similar in both groups. Only the isolate of $C$. glabrata has shown resistant to fluconazole while others were susceptible to Amphotericin B, Voriconazole, Flucytosine, and Caspofungin $^{33}$.

In regard to the frequency of $C$. albicans, our findings were in consistent with the mentioned studies. Our study reported the frequency distribution of $18(62.06 \%)$ C. albicans, 6 (20.68\%) C. glabrata, 4 (13.79\%) C. dubliniensis, and 1 (3.44\%) C. krusei among HIV seropositive women with VVC. The most Candida spp. showed considerable resistant to Amphotericin B and Itraconazole but antifungal susceptibility to Caspofungin. Although, there are similar evidences of Azole antifungal resistance in Candida spp in other studies too, ${ }^{33-35}$ a considerable resistant to Amphotericin B was rarely reported. Hence, our findings emphasize on the use of non- Amphotericin B therapy in addition to non-azole therapy.

According to Badiee et al.'s study in Shiraz, C. albicans (50\%) followed by C. glabrata (21.4\%), C. dubliniensis (13.3\%), C. krusei (9.8\%), C. kefyr (3.1\%), C. parapsilosis (1.6\%), and C. tropicalis ( $0.8 \%)$ were the most prevalent isolated species from mucosal sites in HIV positive patients. All species showed notable sensitivity to amphotericin B, nystatin, voriconazole, ketoconazole and caspofungin while some isolates were resistance to fluconazole and itraconazole. ${ }^{36}$ Their findings were significantly similar to our findings. However, the notable resistant to Amphotericin B was a rare issue in our study.

The frequency of Candida spp colonization in HIV-infected women from United States and Italy was in a higher range (81\%-92\%), ${ }^{37,38}$ compared to our finding (34.11\%). Such variation could be due to the variation in the immune status of people in various regions or other Candidiasis-related-risk factors. So, in the present study we also assessed the association between vulvovaginal candidiasis and some medical variables. In this regard, our findings showed a significant association between VVC and age and pregnancy (PV $<0.05)$ while there was no significant association between CD4 count, the usage history of antibiotics, antiretroviral therapy, birth control methods, history or presence of underlying diseases, and duration of AIDS $(\mathrm{PV}>0.05)$.

\section{Conclusion}

The high occurrence of vulvovaginal Candida spp. (34.11\%) among $H I V$ seropositive women in Ahvaz (Southwest Iran) is notable, with the highest frequency of C. albicans $(62.06 \%)$, followed by C. glabrata (20.68\%) and C. dubliniensis (13.79\%). The considerable resistance of all Candida spp to Amphotericin B and Azole antifungals necessitates more effective treatment programs to reduce the rate of this infection in $H I V$-infected women, particularly in pregnant women.

\section{Funding}

This study was funded by Ahvaz Jundishapur University of Medical Sciences (No: OG-94170).

\section{Ethical approval}

All procedures performed in studies involving human participants were in accordance with the ethical standards of the national research committee and with the 2008 Helsinki declaration and its later amendments or comparable ethical standards. This study was approved by the ethics committee of Ahvaz Jundishapur University of Medical Sciences with Ethical Code: ajums.REC.1396.417.

\section{Authors' contributions}

Study concept and design: Mehran Varnasiri, Shokrolah Salmanzadeh, Ali Zarei Mahmoudabadi.

Sampling and conducting the experiments: Ali Zarei Mahmoudabadi, Marzieh Halvaeezadeh, Simin Taghipour, Shahla Molavi.

Data analysis and interpretation of the results: Mehran Varnasiri, Seyed Mohammad Alavi*, Khojasteh Hoseiny Nezhad, Parastoo Moradi Choghakabodi.

Drafting of the manuscript: Seyed Mohammad Alavi, Khojasteh $\mathrm{H}$. Nezhad, Parastoo Moradi Choghakabodi.

Critical revision of the manuscript: Mehran Varnasiri, Seyed Mohammad Alavi, Khojasteh H. Nezhad, and Parastoo Moradi Choghakabodi.

\section{Declaration of competing interest}

Dr. Mehran Varnaseri and Dr. Seyed Mohammad Alavi has received research grants from Ahvaz Jundishapur University of Medical 


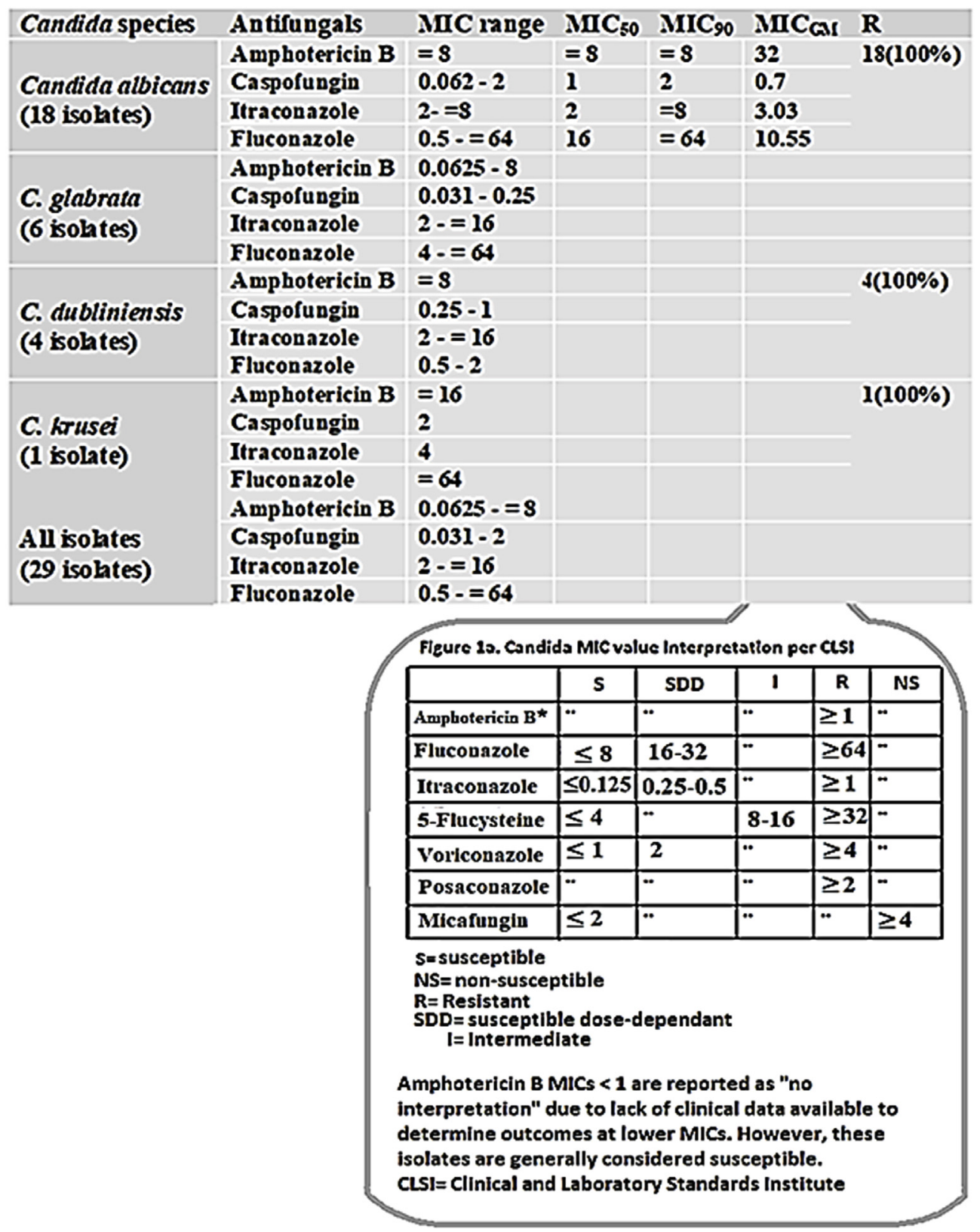

Fig. 1. Antifungal susceptibility of Candida specimens against various antifungals.

Sciences. Other coauthors report no conflicts of interest relevant to this article.

\section{Acknowledgments}

We would like to thank the Health Research Institute, Infectious and Tropical Diseases Research Center, Ahvaz Jundishapur University of Medical Sciences for their support. This study was a MD thesis (Mehran Varnasiri) supported by a grant (No: OG-94170) from the Ahvaz Jundishapur University of Medical Sciences, Ahvaz, Iran.

\section{References}

1. Khan M, Ahmed J, Gul A, Ikram A, Lalani FK. Antifungal susceptibility testing of vulvovaginal Candida species among women attending antenatal clinic in tertiary care hospitals of Peshawar. Infect Drug Resist. 2018;11:447-456.

2. Ohmit S-E, Sobel J-D, Schuman P, et al. Longitudinal study of mucosal Candida species colonization and candidiasis among human immunodeficiency virus (HIV)Seropositive and at-risk HIV-seronegative women. J Infect Dis. 2003;188(1):118-127.
3. Sangeorzan JA, Bradley SF, He X, et al. Epidemiology of oralcandidiasis in HIV-infected patients: colonization, infection, treatment,and emergence of fluconazole resistance. Am J Med. 1994;97 339-3346.3.

4. Fichtenbaum CJ, Powderly WG. Refractory mucosal candidiasis in patients with human immunodeficiency virus infection. Clin Infect Dis. 1998;26 556-5565.4.

5. Moore RD, Chaisson RE. Natural history of opportunistic disease in anHIV-infected urban clinical cohort. Ann Intern Med. 1996;124:633-642.

6. Reed BD, Gorenflo DW, Gillespie BW, Pierson CL, Zazove P. Sexual behaviors and other risk factors for Candida vulvovaginitis. $J$ Wom Health Gend Base Med. 2000;9:645-655

7. Lifson AR, Hilton JF, Westenhouse JL, et al. Time from HIV seroconversion to oral candidiasis or hairy leukoplakia amonghomosexual and bisexual men enrolled in three prospective cohorts. AIDS. 1994:8:73-79.

8. Schuman P, Sobel JD, Ohmit SE, et al. Mucosal candidal colonization and candidiasis in women with or at risk for human immunodeficiency virus infection. HIV Epidemiology Research Study (HERS) Group. Clin Infect Dis. 1998;27:1161-1167.

9. Schuman P, Sobel JD, Ohmit SE, et al. HIV Epidemiology Research Study (HERS) Group. Mucosal candidal colonization and candidiasis in women with or at risk for human immunodeficiency virus infection. Clin Infect Dis. 1998;27:1161-1167.

10. Anwar KP, Malik A, Subhan KH. Profile of candidiasis in HIV infected patients. Iran J Microbiol. 2012;4(4):204-209.

11. Michelet C, Arvieux C, François C, et al. Opportunistic infections occurring during highly active antiretroviral treatment. AIDS. 1998:12:1815-1822.

12. Morgan J. Global trends in candidemia: review of reports from 1995-2005. Curr 
Infect Dis Rep. 2005;7:429-439.

13. Pruthvi BC, Vikram S, Suman SK, Jayaprakash B, Rau NR. 13th International Congress on Infectious Diseases. Spectrum of Clinical Presentation and Opportunistic Infections in HIV. An Indian Scenario; 2006:e484.

14. Nagalingeswaran K, Solomon S, Madhivanan P, et al. Correlation between plasma viral load and CD4 + T cell count to opportunistic infections in persons with HIV in South India. Int Conf AIDS. 2000;9-14:13

15. Singh A, Bairy I, Shivananda PG. Spectrum of opportunistic infections in AIDS cases. Int Conf AIDS. 2003;57:16-21.

16. Pandey S, Sundar S, Hasan H, Shankar R, Singh SP. Clinical profile and opportunistic infection in HIV/AIDS patients attending SS Hospital, Varanasi. Indian J Prev Soc Med. 2008;39:17-20.

17. Nurat AA, Babalola GO, Shittu MO, Tijani MA, Adekola SA. Detection and epidemiology of vulvovaginal candidiasis among asymptomatic pregnant women attending a tertiary hospital in Ogbomoso, Nigeria. Int $J$ Biomed Res. 2015;6(7):518-523.

18. Faraji R, Rahimi MA, Rezvanmadani F, Hashemi M. Prevalence of vaginal candidiasis infection in diabetic women. Afr J Microbiol Res. 2012;6(11):2773-2778.

19. Hedayati MT, Taheri Z, Galinimoghadam T, Aghili SR, Yazdani Cherati J, Mosayebi E. Isolation of different species of Candida in patients with vulvovaginal candidiasis from sari, Iran. Jundishapur J Microbiol. 2015;8(4):e15992.

20. Pouladian S, Movahedi M, Mohammadi R. Clinical and mycological study of vulvovaginal candidiasis (VVC); identification of clinical isolates by polymerase chain reaction-fragment size polymorphyim (PCR-FSP) technique. Arch Clin Infect Dis. 2017;12(2).

21. Mahmoudi Rad M, Zafarghandi A, Amel Zabihi M, Tavallaee M, Mirdamadi Y Identification of Candida species associated with vulvovaginal candidiasis by multiplex PCR. Infect Dis Obstet Gynecol. 2012;2012:872169.

22. Sobel JD, Ohmit SE, Schuman P, et al. The evolution of Candida species and fluconazole susceptibility among oral and vaginal isolates recovered from human immunodeficiency virus (HIV)-seropositive and at-risk HIV-seronegative women. $J$ Infect Dis. 2001;183(2):286-293.

23. Cassone A, Cauda R. Candida and candidiasis in HIV-infected patients: where commensalism, opportunistic behavior and frank pathogenicity lose their borders. AIDS. 2012;26(12):1457-1472.

24. Reference Method for Broth Dilution Abtifungal Suceptibility Testing of Yeasts; Approved Standard-Third Edition. vol. 28, 2008; 2008 M27-A3, 14.

25. Okonkwo N, Umeanaeto P. Prevalence of vaginal candidiasis among pregnant women in Nnewi Town of Anambra State, Nigeria. Afr Res Rev. 2010;4(4):539-548.

26. Kamara P, Hylton-Kong T, Brathwaite A, et al. Vaginal infections in pregnant women in Jamaica: prevalence and risk factors. Int J STD AIDS. 2000;11(8):516-520.

27. Beigi RH, Meyn LA, Moore DM, Krohn MA, Hillier SL. Vaginal yeast colonization in nonpregnant women: a longitudinal study. Obstet Gynecol. 2004;104:926-930 5, pt 1.

28. Nelson M, Wanjiru W, Margaret MW. Prevalence of vaginal candidiasis and determination of the occurrence of Candida species in pregnant women attending the antenatal clinic of Thika District Hospital, Kenya. Open J Med Microbiol. 2013;2013:264-272

29. Spinillo A, Capuzzo E, Nicola S, Baltaro F, Ferrari A, Monaco A. The impact of oral contraception on vulvovaginal candidiasis. Contraception. 1995;51(5):293-297.

30. Dias LB, Melhem MdSC, Szeszs MW, Meirelles Filho J, Hahn RC. Vulvovaginal candidiasis in Mato Grosso, Brazil: pregnancy status, causative species and drugs tests. Braz J Microbiol. 2011;42(4):1300-1307.

31. Sobel JD. Vulvovaginal candidosis. Lancet. 2007;369:1961-1971.

32. Merenstein D, Hu H, Wang C, et al. Colonization by Candida species of the oral and vaginal mucosa in HIV-infected and noninfected women. AIDS Res Hum Retrovir. 2013;29:30-34.

33. Oliveira PM, Mascarenhas RE, Lacroix C, et al. Candida species isolated from the vaginal mucosa of HIV-infected women in Salvador, Bahia, Brazil. Braz J Infect Dis. 2011;15(3):239-244

34. Richter SS, Galask RP, Messer SA, Hollis RJ, Diekema DJ, Pfaller MA. Antifungal susceptibilities of Candida species causing vulvovaginitis and epidemiology of recurrent cases. J Clin Microbiol. 2005;43(5):2155-2162.

35. Yesudhason BL, Mohanra MK. Candida tropicalis as a predominant isolate from clinical specimens and its antifungal susceptibility pattern in a tertiary care hospital in Southern India. J Clin Diagn Res. 2015;9(7):14.

36. Badiee P, Alborzi A, Davarpanah MA, Shakiba E. Distributions and antifungal susceptibility of Candida species from mucosal sites in HIV positive patients. Arch Iran Med. 2010;13(4):282-287.

37. Sobel JD, Ohmit SE, Schuman P, et al. The evolution of Candida spp. and fluconazole susceptibility among oral and vaginal isolates recovered from human immunodeficiency virus (HIV)-seropositive and at-risk HIV-seronegative women. $J$ Infect Dis. 2001;183 286-93.

38. Beltrame A, Matteelli A, Carvalho AC, et al. Vaginal colonization with Candida spp in human immunodeficiency virus-infected women: a cohort study. Int J STD AIDS. $2006 ; 17(4)$ 260-6. 